\title{
Dysphagia risk, low muscle strength and poor cognition predict malnutrition risk in older adults at hospital admission
}

\author{
Idah Chatindiara ${ }^{1 *} \mathbb{D}$, Jacqueline Allen², Amy Popman ${ }^{1}$, Darshan Patel ${ }^{1}$, Marilize Richter ${ }^{1}$, Marlena Kruger ${ }^{1}$ \\ and Carol Wham ${ }^{1}$
}

\begin{abstract}
Background: Malnutrition in patients admitted to hospital may have detrimental effects on recovery and healing. Malnutrition is preceded by a state of malnutrition risk, yet malnutrition risk is often not detected during admission. The aim of the current study was to investigate the magnitude and potential predictors of malnutrition risk in older adults, at hospital admission.

Methods: A cross-sectional was study conducted in 234 older adults (age $\geq 65$ or $\geq 55$ for Māori or Pacific ethnicity) at admission to hospital in Auckland, New Zealand. Assessment of malnutrition risk status was performed using the Mini Nutritional Assessment Short-Form (MNA- ${ }^{-}$SF), dysphagia risk by the Eating Assessment Tool (EAT-10), muscle strength by hand grip strength and cognitive status by the Montreal Cognitive Assessment (MoCA) tool.

Results: Among 234 participants, mean age $83.6 \pm 7.6$ years, 46.6\% were identified as at malnutrition risk and 26.9\% malnourished. After adjusting for age, gender and ethnicity, the study identified [prevalence ratio (95\% confidence interval)] high dysphagia risk [EAT-10 score: $0.98(0.97-0.99)]$, low body mass index [kg/m²: $1.02(1.02-1.03)]$, low muscle strength [hand grip strength, kg: 1.01 (1.00-1.02)] and decline in cognition [MoCA score: 1.01 (1.00-1.02)] as significant predictors of malnutrition risk in older adults at hospital admission.

Conclusion: Among older adults recently admitted to the hospital, almost three-quarters were malnourished or at malnutrition risk. As the majority (88\%) of participants were admitted from the community, this illustrates the need for routine nutrition screening both at hospital admission and in community-dwelling older adults. Factors such as dysphagia, unintentional weight loss, decline in muscle strength, and poor cognition may indicate increased risk of malnutrition.
\end{abstract}

Keywords: Older adults, Hospital malnutrition risk, Dysphagia risk, Muscle strength, MNA ${ }^{\oplus}$-SF

\section{Background}

The current life expectancy at birth (81.7 years) for New Zealand is among the top life expectancies within the Organisation for Economic Co-operation and Development countries [1]. This raises the issue of whether efficient strategies have been put in place to ensure this growth in life expectancy will go along with good quality of life $[2,3]$. Since ageing is a well-documented risk factor for hospitalisation [4-6], there is great need to come up with strategies that help older people to continue living healthily in the

\footnotetext{
* Correspondence: I.Chatindiara@massey.ac.nz

'School of Food and Nutrition, Massey University, Auckland, New Zealand Full list of author information is available at the end of the article
}

community. Although ageing is inexorable, several factors contributing to hospital admission in older adults can be modified, for example, nutrition status. Despite being well documented as a preventable condition, hospital malnutrition is highly prevalent among older adults (20-60\%) [7-9]. It remains unclear whether malnutrition occurs before or after admission since at hospital admission, nutrition screening is not mandatory and is conducted infrequently [10-12].

Malnutrition is preceded by a state of malnutrition risk thus nutrition risk screening may identify those who show nutritional compromise before they reach a state of malnutrition, enabling intervention and prevention. 
Although several international studies assessed malnutrition risk at hospital admission [13-21], the wide range of malnutrition risk prevalence (15-78\%) observed from these studies raises questions. Differences in methodology used to assess malnutrition risk may partly explain the reported discrepancies. Several methods of malnutrition risk screening have been established and the choice of method used should consider participants age and setting [22, 23]. The Mini Nutritional Assessment ShortForm, $\left(\mathrm{MNA}^{\circ}-\mathrm{SF}\right)$ is a validated tool for assessment of malnutrition risk in older adults in multiple settings, including the hospital [24]. The $\mathrm{MNA}^{\circ}$-SF is used globally and has a sensitivity of $89 \%$ and specificity of $82 \%$ for assessing malnutrition risk in older adults [22, 25].

Studies suggest that malnutrition risk factors are multidimensional. These include sociodemographic (e.g. age, gender, living arrangements and employment status), psychological (e.g. individuals' thoughts and feelings), and health status (e.g. functional ability, presence of comorbidities, polypharmacy, impaired chewing and swallowing) [26-30]. Understanding these factors may be helpful for identifying those at malnutrition risk and providing timely preventative interventions. Levels of cognitive impairment often increase with ageing [31], and have the potential to influence nutrition status. Previous studies have reported positive associations between satisfactory dietary intake and better cognition [32]. Furthermore, older adults are more vulnerable to dysphagia (swallowing difficulties) [33], which can lead to malnutrition through decline in food intake. Among healthy community-dwelling older adults, a positive correlation between posterior tongue strength (swallowing strength) and hand grip strength (a measure of muscle strength) was observed [34]. This suggests that loss of muscle strength at one site e.g. hand grip, may be indicative of weakness at other sites e.g. oral cavity. To date, there is limited literature supporting a strong association between tongue strengthening and swallow outcomes. However, among older adults in long term care, reduced tongue strength was found to be associated with observable signs of swallowing difficulty and reduced food consumption [35]. Therefore, exploring associations between muscle strength, dysphagia and malnutrition appears warranted.

In New Zealand, malnutrition risk screening is not mandatory and knowledge of prevalence of malnutrition in older adults is lacking. Using the $\mathrm{MNA}^{\circ}-\mathrm{SF}$, the aim of the current study was to investigate the magnitude and potential predictors of malnutrition risk in older adults, at hospital admission.

\section{Methods}

\section{Setting and sample}

A cross-sectional study was conducted in two hospitals in Auckland, New Zealand. The study included a non- randomised convenience sample of 234 older adults. Recruitment was at hospital admission between July 2014 and September 2015. Ethics approval was granted by the Health and Disability Ethics Committee: Northern A region 14/NTA/70/AMO1.

At hospital admission, participants were provided with an information sheet, the study procedures were explained and written consent was obtained. The inclusion criteria were: age $\geq 65$ or $\geq 55$ for Māori or Pacific ethnicity, admission to hospital rehabilitation wards within the preceding 5 days, able to comprehend study requirements and give consent. A younger age group was included for Māori and Pacific participants as they have a lower life expectancy compared to other ethnicities [36]. Furthermore, findings on health disparities related to ethnicity in New Zealand indicates that Māori and Pacific populations are more likely to biologically age earlier due to poor health $[37,38]$. Participants were excluded if they presented with dysphagia, known gastrointestinal disease, known pre-existing cognitive impairment, malignancy or tracheostomy.

\section{Assessments}

Sociodemographic data including age, gender, ethnicity, marital status, living arrangements, pension-related income and highest education level attained were obtained from participants and clinical notes. Participants were asked if they required help with activities of daily living (ADL) to understand if individuals may be limited in physical functioning. Comorbidities, use of medications and nutritional supplements were evaluated from participant reports and clinical notes. Self-reported dental status was recorded as either dentate (able to chew food without appliances), edentulous (missing teeth contributing to chewing problems and not using dental appliance) or use of dental appliances. Validated tools for screening malnutrition ( $\left.\mathrm{MNA}^{\circ}-\mathrm{SF}\right)$, dysphagia risk (Eating Assessment Tool-10 (EAT-10)) and cognitive status (Montreal Cognitive Assessment (MoCA)) in older adults were used. Furthermore, physical measurements specifically weight, height, body mass index (BMI) and muscle strength were done.

\section{Malnutrition risk status}

The MNA ${ }^{\circ}-\mathrm{SF}$ is comprised of six items that assess food intake, weight loss, mobility, psychological stress, neuropsychological problems, and BMI. Using the previously validated $\mathrm{MNA}^{\circ}$-SF cut-off points; nutrition status was defined as the state of being either well-nourished $\left(\mathrm{MNA}^{\circ}\right.$-SF score $\geq 12$ ), at malnutrition risk ( $\mathrm{MNA}^{\circ}$-SF score $\left.8-11\right)$ or malnourished ( $\mathrm{MNA}^{\circ}$-SF score 0-7). Furthermore, malnutrition risk status was categorised as: 'at risk' $\left(\mathrm{MNA}^{\circ}-\mathrm{SF}\right.$ score $\left.\leq 11\right)$ and 'not at risk' $\left(\mathrm{MNA}^{\circ}-\mathrm{SF}\right.$ score $\geq 12$ ). Lower $\mathrm{MNA}^{\circ}$-SF score indicate increased 
malnutrition risk. All assessments were performed per the $\mathrm{MNA}^{\circ}$-SF user guide [24].

\section{Dysphagia risk}

EAT-10, a self-reported validated questionnaire that assesses perception of swallowing difficulty was used to evaluate dysphagia risk. Increase in the EAT-10 score is indicative of increased dysphagia risk or swallowing difficulties, and an EAT-10 score $\geq 3$ is suggestive of dysphagia [39].

\section{Cognitive status}

Cognitive status was determined using the MoCA tool. This tool is a multi-domain test which enables detection of mild cognitive impairment through several assessments including memory, attention, conceptual thinking and orientation. Low MoCA scores indicate decline in cognitive status and a score $\leq 26$ indicates cognitive impairment [40].

\section{Muscle strength}

Muscle strength was assessed using hand grip strength [41]. A hand dynamometer (Jamar Hydraulic Dynamometer, model \#5030 J1, Sammons Preston, USA) was used. The measurement procedure used conformed to the standard approved by the American Society of Hand Therapists [42]. The mean of six measurements, three from each hand was recorded. In participants $(n=8)$ who could only provide measurements from one hand, a mean of three measurements was used from the dominant hand [42, 43].

\section{Statistical analysis}

The main outcome measure was malnutrition risk indicator score $\left(\mathrm{MNA}^{\oplus}-\mathrm{SF}\right)$. Continuous data was assessed for normality using Shapiro Wilcoxon tests and through visual analysis of the normality plots. Descriptive statistics for normally distributed data were presented as mean \pm standard deviation (SD) and categorical data were presented as frequencies and percentages. Chisquare test of independence or Fisher exact tests was used to analyse the association between participants' demographic characteristics and malnutrition status. As the prevalence of malnutrition risk was higher than $10 \%$, Poisson regression with robust variance estimation was used to quantify the magnitude of the associations between potential predictors and malnutrition risk (MNA ${ }^{\circ}$-SF score) [44]. Each characteristic was added to the Poisson regression model adjusting for the major non-modifiable human factors (age, gender and ethnicity), medications and comorbidities, as these factors are related to malnutrition risk. The magnitudes of associations were reported as prevalence ratio (PR) and 95\% confidence interval $(95 \% \mathrm{CI})$. Results were considered significant at $p<0.05$. All statistical analyses were done using IBM SPSS Statistics version 24.

\section{Results}

Study population and prevalence of malnutrition risk Malnutrition risk assessment was completed in 234 participants aged (mean years \pm SD) $83.6 \pm 7.6$, of whom $40.6 \%$ were women. Eighty-eight percent of participants were admitted from the community and $41 \%$ had a BMI less than $23 \mathrm{~kg} / \mathrm{m}^{2}$, which is below the proposed healthy cut-off point $\left(B M I \geq 23 \mathrm{~kg} / \mathrm{m}^{2}\right)$ for older adults [45]. Data collection was conducted within 5 days of hospital admission, regardless of admission reason. No adverse events were encountered during the study data collection. Table 1 shows the participants characteristics by nutrition status.

The study identified 109 participants (46.6\%) at malnutrition risk $\left(\mathrm{MNA}^{\circ}\right.$-SF score $\left.8-11\right)$ and 63 participants (26.9\%) as malnourished ( $\mathrm{MNA}^{\circ}$-SF score $\left.12-14\right)$.

\section{Physical and health predictors of malnutrition risk}

Table 2 summarises the descriptive data for predictive factors evaluated in relation to malnutrition risk, and the respective magnitudes [PR $(95 \% \mathrm{CI})]$ of association with malnutrition observed. From the descriptive data, participants showed a general decline in physical and health status. Only a third maintained full dentition and an increased risk of swallowing problems was suggested by the elevated mean EAT-10 score of $3.0( \pm 5.8)$. A majority of the participants had reduced muscle strength (hand grip), reduced cognitive functioning scores, required daily help with ADL and had approximately six comorbidities. Hypertension (and other vascular diseases), osteoporosis and chronic obstructive pulmonary disease were the most frequently observed conditions.

After adjusting for age, gender and ethnicity, analysis from Poisson regression with robust variance estimation identified [PR (95\% CI)] high dysphagia risk [EAT-10 score: 0.98 (0.97-0.99)], low muscle strength [hand grip strength (kg): $1.01(1.00-1.02)]$, low BMI $\left(\mathrm{kg} / \mathrm{m}^{2}\right)$ [1.02 (1.02-1.03)] and decline in cognition [MoCA score: 1.01 (1.00-1.02)] as significant predictors of malnutrition risk in older adults at hospital admission (Table 2).

\section{Discussion}

This is the first study in New Zealand to assess prevalence and predictors of malnutrition risk in older adults at hospital admission. Almost three-quarters were either malnourished $(26.9 \%)$ or at malnutrition risk (46.6\%), congruent with previously published international findings (15-78\%) [13-21]. Dysphagia risk, low BMI, decreased muscle strength and cognitive decline, were identified as statistically significant predictors of malnutrition risk, at hospital admission. As $88 \%$ of participants 
Table 1 Participants' demographic characteristics by malnutrition status: MNA ${ }^{\circledR}-\mathrm{SF}$

\begin{tabular}{|c|c|c|c|c|}
\hline \multirow[t]{3}{*}{ Characteristics } & \multirow{3}{*}{$\begin{array}{l}{ }^{*} \text { Total } \\
n(\%)\end{array}$} & \multicolumn{3}{|c|}{ Malnutrition status } \\
\hline & & Well-nourished & At malnutrition risk & Malnourished \\
\hline & & $n(\%)$ & $n(\%)$ & $n(\%)$ \\
\hline All participants' & $234(100)$ & $62(26.5)$ & $109(46.6)$ & $63(26.9)$ \\
\hline \multicolumn{5}{|l|}{ Admission source } \\
\hline - Community & $156(88.1)$ & $46(29.5)$ & $70(44.9)$ & $40(25.6)$ \\
\hline - Aged care ${ }^{a}$ & $21(11.9)$ & $5(23.8)$ & $7(33.3)$ & $9(42.9)$ \\
\hline \multicolumn{5}{|l|}{ Age } \\
\hline$-<85$ years & $122(52.1)$ & $32(26.2)$ & $58(47.5)$ & $32(26.2)$ \\
\hline - $\geq 85$ years & $112(47.9)$ & $30(26.8)$ & $51(45.5)$ & $31(27.7)$ \\
\hline \multicolumn{5}{|l|}{ BMl } \\
\hline$-<23 \mathrm{~kg} / \mathrm{m}^{2}$ & $95(40.6)^{* *}$ & $9(9.5)$ & $45(47.4)$ & $41(43.2)$ \\
\hline$-\geq 23 \mathrm{~kg} / \mathrm{m}^{2}$ & $139(59.4)$ & $53(38.1)$ & $64(46.0)$ & $22(15.8)$ \\
\hline \multicolumn{5}{|l|}{ Gender } \\
\hline - Men & 95 (40.6) & $25(26.3)$ & $46(48.4)$ & $24(25.3)$ \\
\hline - Women & $139(59.4)$ & $37(26.6)$ & $63(45.3)$ & $39(28.1)$ \\
\hline \multicolumn{5}{|l|}{ Marital status } \\
\hline - Married/partnered & 95 (40.6) & $26(27.4)$ & $42(44.2)$ & $27(28.4)$ \\
\hline - Single & $139(59.4)$ & $36(25.9)$ & $67(48.2)$ & $36(25.9)$ \\
\hline \multicolumn{5}{|l|}{ Ethnicity } \\
\hline - All other ethnicities ${ }^{c}$ & $87(37.2)$ & $21(24.1)$ & $38(43.7)$ & $28(32.2)$ \\
\hline - New Zealand European & $147(62.8)$ & $41(27.9)$ & $71(48.3)$ & $35(23.8)$ \\
\hline \multicolumn{5}{|l|}{ Living arrangements } \\
\hline - Living alone & $104(44.4)$ & $31(29.8)$ & $49(47.1)$ & $24(23.1)$ \\
\hline - Living with partner only & $73(31.2)$ & $21(28.8)$ & $32(43.8)$ & $20(27.4)$ \\
\hline - Living with others & $57(24.4)$ & $10(17.5)$ & $28(49.1)$ & $19(33.3)$ \\
\hline \multicolumn{5}{|l|}{ Income source } \\
\hline - Pension only income & $152(65.0)$ & $37(24.3)$ & $69(45.4)$ & $46(30.3)$ \\
\hline - Pension plus other income & $82(35.0)$ & $25(30.5)$ & $40(48.8)$ & $17(20.7)$ \\
\hline \multicolumn{5}{|l|}{ Education } \\
\hline - Primary & $52(22.2)$ & $11(21.2)$ & $28(53.8)$ & $13(25.0)$ \\
\hline - Secondary & $144(61.5)$ & $38(26.4)$ & $62(43.1)$ & $44(30.6)$ \\
\hline - Tertiary & $38(16.2)$ & $13(34.2)$ & $19(50.0)$ & $6(15.8)$ \\
\hline
\end{tabular}

$B M I$ body mass index, $M N A^{\oplus}$-SF Mini Nutritional Assessment Short-Form

*The percentages (\%) in the total column are percentage total. All other percentages are percentage total within a category. All values are reported after rounding up to 1 decimal place, therefore percentages add up to $100 \pm 0.1^{* *}$ Only BMI was statistically significant across nutrition status groups: $p<0.05$ Chi-square test of independence

${ }^{a}$ Aged care: admitted from long term residential care $(n=16)$ or private hospital interim care $(n=5)$

${ }^{\mathrm{b}}$ Marital status single: widowed $n=110$, divorced $n=15$, single/never married $n=14$

'Other ethnicities: Māori $n=6$, Pacific $n=6$, others $n=75$

were admitted from the community, this suggests that the high prevalence of hospital malnutrition may be a result of unrecognised community malnutrition; hence routine screening is essential on hospital admission. Furthermore, it suggests that community based assessment and intervention may be important for identifying at-risk adults and providing early supportive measures.
The prevalence of malnutrition (26.9\%) observed in this study is congruent with previously reported hospital malnutrition prevalence $(20-60 \%)$ internationally [7-9]. This suggests that both malnutrition risk and malnutrition are present in older adults prior to hospital admission. This study is an extension of our pilot investigation that reported malnutrition risk prevalence in adults $(n=88)$ of 
Table 2 Physical and health predictors of malnutrition risk indicator score (MNA $\left.{ }^{\oplus}-\mathrm{SF}\right)$

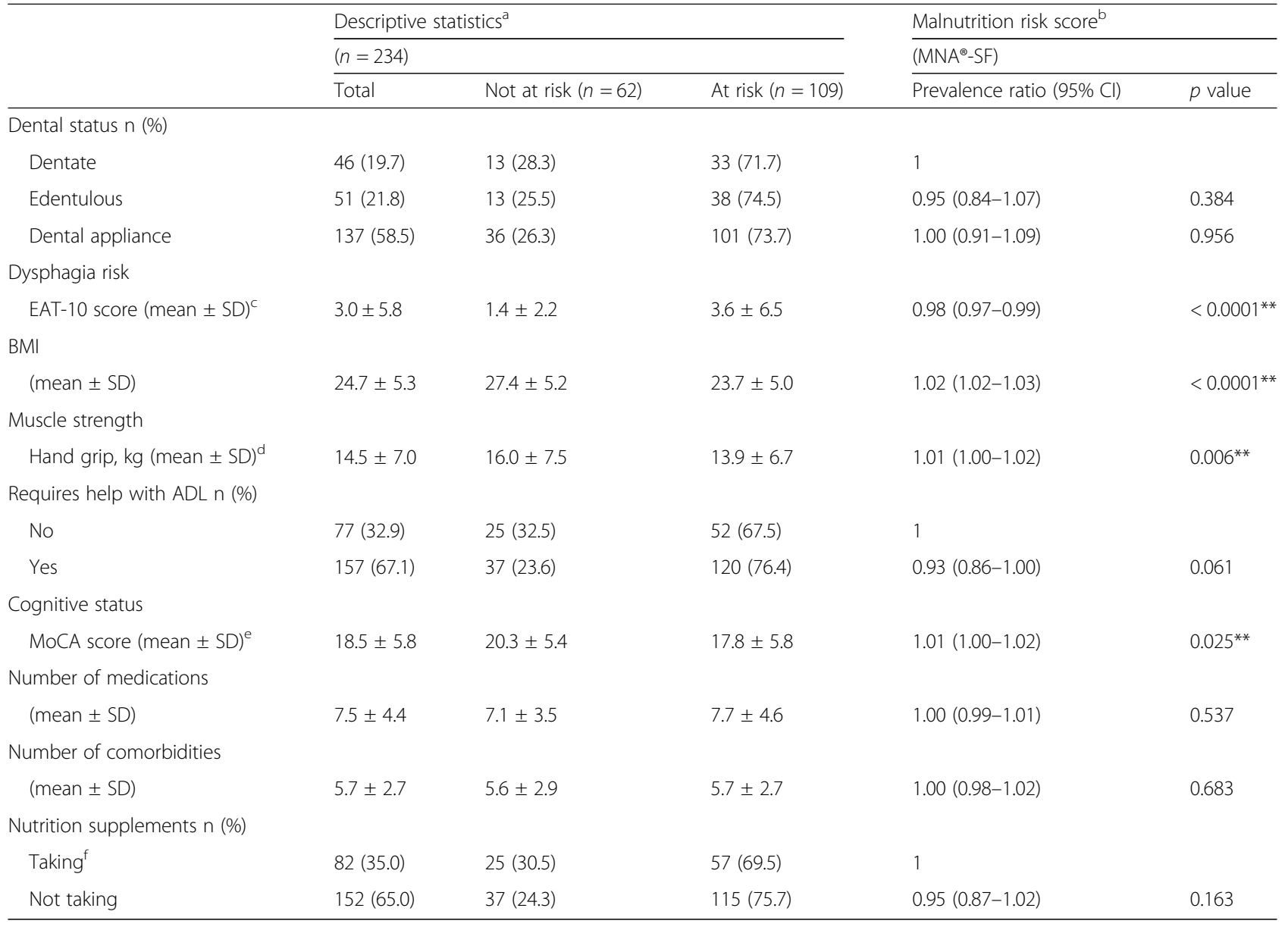

$M N A^{\oplus}$-SF Mini Nutritional Assessment Short-Form, EAT-10 Eating Assessment Tool, BMI body mass index, $A D L$ activities of daily living, MoCA Montreal Cognitive Assessment

${ }^{a}$ Descriptive statistics for continuous data were presented as mean \pm standard deviation (SD) and categorical data presented as frequencies ( $\mathrm{n}$ ) and percentages (\%)

${ }^{b}$ Poisson regression with robust variance estimation. Each parameter was adjusted for age, gender, ethnicity, number of comorbidities and medications; PR (95\%

Cl) ${ }^{* *} p$ value significant at $p<0.05$

"Missing data $\mathrm{n}=2$

dMissing data $n=66$

e Missing data $n=69$

${ }^{f}$ Average number of supplements taken $n=1$

advanced age (85+ years), wherein a similar prevalence of malnutrition risk was observed [46]. Previously, malnutrition has been identified in New Zealand older adults $(n=$ 55 ) hospitalised for hip fracture; where $42 \%$ were found to have at least two indices of protein energy malnutrition as evidenced by low triceps skinfold thickness, reduced midupper arm circumference, and low serum pre-albumin [47]. Moreover, using the $\mathrm{MNA}^{\oplus}-\mathrm{SF}$, a New Zealand hospital audit of older adults $(n=72)$ found $24 \%$ were malnourished and $44 \%$ were at nutrition risk [48]. The insights from the aforementioned reports and findings from our study suggest the need for routine screening at hospital admission in New Zealand.

Previous studies reported chewing and swallowing problems as common phenomena in older people, which may lead to decline in food intake [49-51]. Reduced food intake is one of the main causes of malnutrition. Thus, understanding both dental status and dysphagia risk may be helpful in devising strategies intended to prevent malnutrition. In contrast to previous studies [52, 53], no significant association between dental status and malnutrition risk status was observed in the current study. However, as expected, we observed that with increasing risk of dysphagia (increasing EAT-10 scores), the prevalence ratio of malnutrition risk also increased. This concurs with findings of a previous study that assessed the association between swallowing difficulties and hospital malnutrition [28]. In the current study, 22\% of the participants demonstrated EAT-10 scores $\geq 3$ which suggests that in older people who are malnourished or at malnutrition risk, consideration of swallow assessment would be prudent. This may identify contributory 
problems and define treatment targets. Furthermore, for treatment of malnutrition, it may be helpful if future studies investigate the clinically relevant cut-off points for the EAT-10 associated with malnutrition risk, since an EAT10 score $\geq 3$ is merely suggestive of dysphagia [39].

Greater muscle strength as indicated by greater hand grip strength was associated with lower malnutrition risk. Similar findings were observed in an Australian study that explored the potential of hand grip strength to independently predict nutrition status in a hospital population [54]. A Canadian study that assessed malnutrition at hospital admission observed hand grip strength to be an independent factor associated with hospital stay [14]. Several factors explain the occurrence of poor muscle strength in older adults and how they may create a vicious cycle resulting in malnutrition. Reduced physical activity and functional decline commonly observed in older adults contribute to muscle atrophy and poor muscle strength [55-57]. In addition, malnutrition promotes body weight loss (low BMI), particularly muscle mass loss, including the deglutitive muscles, which consequently increases swallowing problems and hence malnutrition risk $[58,59]$.

Muscle weakness and low BMI often occur together in undernourished people [60,61]. Our study supports this association, as a positive correlation $(r=0.236, p<0.046)$ between BMI and muscle strength was observed. BMI is an integral part of the $\mathrm{MNA}^{\circ}$-SF and in older adults high BMI values are associated with low malnutrition risk [28, 62], better functional status [63] and may be protective against mortality [62, 64, 65]. Accordingly, a BMI $\geq 23 \mathrm{~kg} / \mathrm{m}^{2}$ has been proposed as the healthy cut-off point for older adults [45]. In the current study, $41 \%$ of the participants had a $\mathrm{BMI}<23 \mathrm{~kg} / \mathrm{m}^{2}$ which may explain the high prevalence of malnutrition risk observed.

Two-thirds of participants required daily help with various tasks such as cooking, cleaning, showering and dressing. This may suggest loss of physical function among these participants which may contribute to the low muscle strength and high malnutrition risk observed. Previous studies reported that malnutrition risk is higher in older adults who rely on others for assistance with daily activities like grocery shopping and cooking $[8,14,26]$. Although not statistically significant ( $p=0.061)$, our data demonstrated an increased risk of malnutrition among older adults who required help with ADL. Whether this incremental decrease in nutrition status can be detected clinically is not clear from this study.

Medications and comorbidities are well documented factors associated with malnutrition $[8,26]$. In the current study, on average each participant had six comorbidities and was taking seven medications. Although these factors were not significantly associated with malnutrition risk in this study, they may contribute to decreased physical activity, decline in muscle mass and consequently poor muscle strength. These factors negatively influence malnutrition risk [58, 59]. Two-thirds $(65 \%)$ of the participants did not take any nutritional supplements and among those who took supplements, on average only one supplement was taken. This may explain why taking nutrition supplements did not impact malnutrition risk. A recent study in New Zealand observed low micronutrient intake in older adults and noted that with some of the micronutrients, those who took nutritional supplements were less likely to be nutrient deficient [66]. Further education in this area may assist older adults in determining whether supplementation is appropriate.

In agreement with previous studies [26], malnutrition risk was associated with decline in cognitive status. Cognitive impairment is associated loss of independence which may negatively affect older adults' lifestyle, specifically food intake and physical activity. Malnutrition and cognitive impairment have been reported as predictors of allcause mortality among hospitalised adults ( $>70$ years) [67]. In the current study, $62 \%$ of the participants indicated some level of cognitive impairment (MoCA score $\leq 26)$. Although about a quarter of the participants declined taking the MoCA test, the high prevalence of cognitive impairment among the test takers suggests the importance of performing cognitive assessments in older adults.

The strengths of this study include the use of validated screening tool $\mathrm{MNA}^{\circ}$-SF and the use of a critical time point for data collection i.e. at hospital admission. These factors enable understanding of malnutrition risk in older New Zealanders at hospital admission, which provides helpful insights towards promoting good nutritional status not only during hospitalisation but also prior in the community and after discharge. However, the use of an observational study design hinders us from determining causality. Additionally, investigations were done using a convenience sample $(n=234)$, which limits generalisability of the findings. Hand grip strength and cognitive status assessments were undertaken with about three-quarters of the participants, therefore interpretation of these results should be made with caution, as the missing data may have introduced bias in our findings. Overall, the participants had low MoCA scores which may have influenced the malnutrition risk outcome, since some of the MNA ${ }^{\circ} \mathrm{SF}$ items rely on memory. Due to poor physical strength and/or presence of metals in the body, only 16 participants completed muscle mass measurement using bio-impedance analysis scale, thus we could not report muscle mass findings. As significant changes in body composition occur with ageing, inability to assess muscle mass in this study limited our ability to understand the impact of body composition on malnutrition 
risk. To understand participants' general health status, the current study recorded comorbidities and medications taken. However, the study did not capture reason for admission, which would have helped clarify participants' health upon admission.

\section{Conclusion}

Among older adults recently admitted to hospital rehabilitation wards, almost three-quarters were malnourished or at malnutrition risk. This illustrates the need for timely screening, as early identification is one of the most important and effective ways to prevent and reduce the prevalence of malnutrition and malnutrition risk. As $88 \%$ of the participants were admitted from the community, this illustrates the need for routine nutrition screening both at hospital admission and in community-dwelling older adults. Routine screening, especially at hospital admission, would identify those that may benefit from intervention or nutritional support during hospitalisation. Interventions that focus on increasing muscle strength and/or preventing dysphagia risk, while considering the cognitive functioning of older adults may reduce malnutrition risk. This cross-sectional study indicates the need for robust intervention studies to investigate the efficacy of prevention or reversal of malnutrition risk factors on nutritional status.

\section{Abbreviations}

ADL: Activities of daily living; BMl: Body mass index; Cl: Confidence interval; EAT-10: Eating Assessment Tool; MNA ${ }^{\oplus}$-SF: Mini Nutritional Assessment ShortForm; MoCA: Montreal Cognitive Assessment; PR: Prevalence ratio;

SD: Standard deviation

\section{Acknowledgements}

The authors greatly appreciate the contribution of Teresa Stanbrook, Waitemata DHB and all the participants who made the data collection process possible.

\section{Funding}

This research received no specific grant from any funding agency.

\section{Availability of data and materials}

All original data is available at Massey University College of Health, Auckland campus, New Zealand. The corresponding author can share this data upon request.

\section{Authors' contributions \\ IC wrote the manuscript, conducted statistical analysis and interpretation of the data. CW and JA designed the study and participated in manuscript preparation AP and DP collected the data participated in pre-final manuscript editing. MR and MK provided input for statistical design, analysis and interpretation. All authors participated in the manuscript proof reading and approved the final manuscript.}

\section{Ethics approval and consent to participate}

Ethics approval was granted by the Health and Disability Ethics Committee: Northern A region 14/NTA/70/AMO1. Participants were provided with the study information and the study procedures were explained. Written consent was obtained before data collection.

\section{Consent for publication}

Not applicable.

\section{Competing interests}

The authors declare that they have no competing interests.

\section{Publisher's Note}

Springer Nature remains neutral with regard to jurisdictional claims in published maps and institutional affiliations.

\section{Author details}

${ }^{1}$ School of Food and Nutrition, Massey University, Auckland, New Zealand.

${ }^{2}$ Department of Surgery, University of Auckland, Auckland, New Zealand.

Received: 11 October 2017 Accepted: 16 March 2018

Published online: 21 March 2018

\section{References}

1. OECD. Life expectancy at birth (indicator). 2018 [Accessed on 22 Jan 2018]; Available from: https://data.oecd.org/healthstat/life-expectancy-at-birth.htm - indicator-chart

2. Christensen $\mathrm{K}$, et al. Ageing populations: the challenges ahead. Lancet. 2009:374(9696):1196-208.

3. Dalziel L. The New Zealand positive ageing strategy: Towards a society for all ages. Wellington: Ministry of Social Policy; 2001.

4. Boult $C$, et al. Screening elders for risk of hospital admission. J Am Geriatr Soc. 1993:41(8):811-7.

5. Dorr DA, et al. Use of health-related, quality-of-life metrics to predict mortality and hospitalizations in community-dwelling seniors. J Am Geriatr Soc. 2006;54(4):667-73.

6. Inouye SK, et al. Risk factors for hospitalization among community-dwelling primary care older patients: development and validation of a predictive model. Med Care. 2008:46(7):726-31.

7. Barker LA, Gout BS, Crowe TC. Hospital malnutrition: prevalence, identification and impact on patients and the healthcare system. Int J Environ Res Public Health. 2011:8(2):514-27.

8. Agarwal $\mathrm{E}$, et al. Malnutrition in the elderly: a narrative review. Maturitas. 2013;76(4):296-302.

9. Kaiser MJ, et al. Frequency of malnutrition in older adults: a multinational perspective using the mini nutritional assessment. J Am Geriatr Soc. 2010; 58(9):1734-8.

10. McWhirter JP, Pennington CR. Incidence and recognition of malnutrition in hospital. BMJ. 1994;308(6934):945-8

11. Tappenden KA, et al. Critical role of nutrition in improving quality of care: an interdisciplinary call to action to address adult hospital malnutrition. J Acad Nutr Diet. 2013;113(9):1219-37.

12. Starr KNP, McDonald SR, Bales CW. Nutritional vulnerability in older adults: a continuum of concerns. Curr Nutr Rep. 2015;4(2):176-84.

13. Pablo AR, Izaga MA, Alday LA. Assessment of nutritional status on hospital admission: nutritional scores. Eur J Clin Nutr. 2003;57(7):824-31.

14. Allard JP, et al. Malnutrition at hospital admission — contributors and effect on length of stay: a prospective cohort study from the Canadian Malnutrition Task Force. J Parenter Enter Nutr. 2016:40(4):487-97.

15. Imoberdorf $\mathrm{R}$, et al. Prevalence of undernutrition on admission to Swiss hospitals. Clin Nutr. 2010;29(1):38-41.

16. Amaral TF, et al. The economic impact of disease-related malnutrition at hospital admission. Clin Nutr. 2007;26(6):778-84.

17. Korfalı G, et al. Nutritional risk of hospitalized patients in Turkey. Clin Nutr. 2009;28(5):533-7.

18. Planas $\mathrm{M}$, et al. Nutritional status among adult patients admitted to an university-affiliated hospital in Spain at the time of genoma. Clin Nutr. 2004; 23(5):1016-24.

19. Rasmussen $\mathrm{HH}$, et al. Prevalence of patients at nutritional risk in Danish hospitals. Clin Nutr. 2004;23(5):1009-15.

20. Amaral TF, et al. Undernutrition and associated factors among hospitalized patients. Clin Nutr. 2010;29(5):580-5.

21. Kyle $\mathrm{U}$, et al. Prevalence of malnutrition in 1760 patients at hospital admission: a controlled population study of body composition. Clin Nutr. 2003;22(5):473-81.

22. Bales CW, Locher JL, Saltzman E, eds. Handbook of clinical nutrition and aging. Third ed. New York: Springer Science+Business Media; 2016. p. 35-53.

23. Guaitoli PR, Jansma EP, de Vet HC. Nutrition screening tools: Does one size fit all? A systematic review of screening tools for the hospital setting. Clin Nutr. 2014;33(1):39-58. 
24. Rubenstein $L Z$, et al. Screening for undernutrition in geriatric practice developing the Short-Form Mini-Nutritional Assessment (MNA-SF). J Gerontol Ser A Biol Med Sci. 2001;56(6):M366-72.

25. Mueller C, Compher C, Ellen DM. ASPEN clinical guidelines nutrition screening, assessment, and intervention in adults. J Parenter Enter Nutr. 2011;35(1):16-24.

26. Moreira NCF, et al. Risk factors for malnutrition in older adults: a systematic review of the literature based on longitudinal data. Advances in Nutrition: An International Review Journal. 2016;7(3):507-22.

27. Tamura BK, et al. Factors associated with weight loss, low BMl, and malnutrition among nursing home patients: a systematic review of the literature. J Am Med Dir Assoc. 2013;14(9):649-55.

28. Vanderwee $\mathrm{K}$, et al. Malnutrition and associated factors in elderly hospital patients: a Belgian cross-sectional, multi-centre study. Clin Nutr. 2010;29(4): 469-76

29. Pirlich M, et al. The German hospital malnutrition study. Clin Nutr. 2006; 25(4):563-72.

30. Wham C, Redwood K, Kerse N. Validation of the nutrition screening tool 'seniors in the community: risk evaluation for eating and nutrition, version II' among octogenarians. J Nutr Health Aging. 2014;18(1):39-43.

31. Glisky EL. Changes in cognitive function in human aging, Brain aging: models, methods, and mechanisms; 2007. p. 3-20.

32. Ortega RM, et al. Dietary intake and cognitive function in a group of elderly people. Am J Clin Nutr. 1997;66(4):803-9.

33. Humbert IA, Robbins J. Dysphagia in the elderly. Phys Med Rehabil Clin N Am. 2008;19(4):853-66.

34. Butler SG, et al. The relationship of aspiration status with tongue and handgrip strength in healthy older adults. J Gerontol Ser A Biol Med Sci. 2011;66(4):452-8.

35. Namasivayam AM, Steele $\mathrm{CM}$, Keller $\mathrm{H}$. The effect of tongue strength on meal consumption in long term care. Clin Nutr. 2016;35(5):1078-83.

36. Statistics New Zealand. Population. 2016 [Accessed on 22 Nov 2017]; Available from: http://www.stats.govt.nz/browse_for_stats/population.aspx.

37. Bramley, D., et al., Indigenous disparities in disease-specific mortality, a cross-country comparison: New Zealand, Australia, Canada, and the United States. 2004.

38. Dyall L. Dementia: continuation of health and ethnic inequalities in New Zealand. N Z Med J (Online). 2014;127(1389):68-80.

39. Belafsky PC, et al. Validity and reliability of the eating assessment tool (EAT10). Ann Otol Rhinol Laryngol. 2008;117(12):919-24.

40. Nasreddine ZS, et al. The Montreal Cognitive Assessment, MoCA: a brief screening tool for mild cognitive impairment. J Am Geriatr Soc. 2005:53(4):695-9.

41. Cruz-Jentoft AJ, et al. Sarcopenia: European consensus on definition and diagnosis report of the European Working Group on Sarcopenia in Older People. Age Ageing. 2010;39(4):412-23.

42. Roberts $\mathrm{HC}$, et al. A review of the measurement of grip strength in clinical and epidemiological studies: towards a standardised approach. Age Ageing. 2011:40(4):423-9.

43. Coldham F, Lewis J, Lee $\mathrm{H}$. The reliability of one vs. three grip trials in symptomatic and asymptomatic subjects. J Hand Ther. 2006;19(3):318-27.

44. Barros AJ, Hirakata VN. Alternatives for logistic regression in cross-sectional studies: an empirical comparison of models that directly estimate the prevalence ratio. BMC Med Res Methodol. 2003;3(1):21.

45. Winter JE, et al. BMI and all-cause mortality in older adults: a meta-analysis. Am J Clin Nutr. 2014:99(4):875-90.

46. Popman A, et al. High nutrition risk is associated with higher risk of dysphagia in advanced age adults newly admitted to hospital. Nutr Diet. 2017;75(1):52-8.

47. Hanger $\mathrm{H}$, et al. The prevalence of malnutrition in elderly hip fracture patients. N Z Med J. 1999;112(1084):88-90

48. Van Lill S. Audit on the nutritional status of patients over 65 years in the AT\&R wards, Middlemore Hospital. In: Proceedings of the New Zealand Dietetic Association Inc. Conference; 2002.

49. Petersen PE, Yamamoto T. Improving the oral health of older people: the approach of the WHO Global Oral Health Programme. Community Dent Oral Epidemiol. 2005;33(2):81-92.

50. Thomson WM, Ma S. An ageing population poses dental challenges. Singap Dent J. 2014;35:3-8.

51. Hickson M. Malnutrition and ageing. Postgrad Med J. 2006:82(963):2-8.

52. Poisson $P$, et al. Relationships between oral health, dysphagia and undernutrition in hospitalised elderly patients. Gerodontology. 2016; 33(2):161-8.
53. Saarela RK, et al. Dentition, nutritional status and adequacy of dietary intake among older residents in assisted living facilities. Gerodontology. 2016;33(2):225-32.

54. Flood A, et al. The use of hand grip strength as a predictor of nutrition status in hospital patients. Clin Nutr. 2014;33(1):106-14.

55. Baumgartner RN, et al. Predictors of skeletal muscle mass in elderly men and women. Mech Ageing Dev. 1999;107(2):123-36.

56. Sakuma K, Yamaguchi A. Sarcopenia and age-related endocrine function. Int J Endocrinol. 2012;2012:127362. https://doi.org/10.1155/2012/127362.

57. Colón-Emeric CS, et al. Functional decline in older adults. Am Fam Physician. 2013:88(6):388-94

58. Visvanathan R. Under-nutrition in older people: a serious and growing global problem! J Postgrad Med. 2003;49(4):352-60.

59. Lee JS, Frongillo EA Jr. Factors associated with food insecurity among US elderly persons importance of functional impairments. J Gerontol Ser B Psychol Sci Soc Sci. 2001;56(2):S94-9.

60. Norman $\mathrm{K}$, et al. The subjective global assessment reliably identifies malnutrition-related muscle dysfunction. Clin Nutr. 2005;24(1):143-50.

61. Norman K, et al. Hand grip strength: outcome predictor and marker of nutritional status. Clin Nutr. 2011;30(2):135-42.

62. Hogue CW, et al. The impact of obesity on outcomes after critical illness: a meta-analysis. Intensive Care Med. 2009;35(7):1152-70.

63. Bahat $\mathrm{G}$, et al. Which body mass index (BMI) is better in the elderly for functional status? Arch Gerontol Geriatr. 2012;54(1):78-81.

64. Donini LM, et al. A systematic review of the literature concerning the relationship between obesity and mortality in the elderly. J Nutr Health Aging. 2012;16(1):89-98

65. Thinggaard $\mathrm{M}$, et al. Is the relationship between $\mathrm{BMI}$ and mortality increasingly U-shaped with advancing age? A 10-year follow-up of persons aged 70-95 years. J Gerontol Ser A Biol Med Sci. 2010;65(5):526-31.

66. Wham C, et al. Micronutrient intake in advanced age: Te Puāwaitanga o Ngā Tapuwae Kia ora Tonu, life and living in advanced age: a cohort study in New Zealand (LiLACS NZ). Br J Nutr. 2016:116(10):1754-69.

67. Farid $\mathrm{K}$, et al. Cognitive impairment and malnutrition, predictors of all-cause mortality in hospitalized elderly subjects with cardiovascular disease. Arch Cardiovasc Dis. 2013;106(4):188-95.

\section{Submit your next manuscript to BioMed Central and we will help you at every step:}

- We accept pre-submission inquiries

- Our selector tool helps you to find the most relevant journal

- We provide round the clock customer support

- Convenient online submission

- Thorough peer review

- Inclusion in PubMed and all major indexing services

- Maximum visibility for your research

Submit your manuscript at www.biomedcentral.com/submit 